1 Becker KL, Winnacker JL, Matthews MJ, Higgins GA. Gynecomastia and hyperthyroidism. An endocrine and histological investigation. F Clin Endocrinol Metab 1968;28:277-85.

2 Ashkar FS, Smoak WM III, Gilson AJ, Miller R. Gynecomastia and mastoplasia in Graves' disease. Metabolism 1970;19:946-51.

3 Cheah JS.Gynaecomastia in hyperthyroidism. Singapore Med f 1971;12:241-3.

4 MuthusamyE. Hyperthyroidism with gynaecomastia, galactorrhoea and periodic paralysis. Singapore Med f 1991;32:371-2.

5 Tan TT, Ng ML, Wu LL, Khalid BA. Hyperthyroid Graves disease. A 5 year retrospective study. Med F Malaysia 1989 44:224-30.
6 Nomura K, Suzuki H, Saji M, et al. High serum progesterone in hyperthyroid men with Graves' disease. 7 Clin Endoone in hyperthyroid men with

7 Glass AR. Gynecomastia. Endocrinol Metab Clin North Am 1994;23:825-37.

8 Chopra IJ, Tulchinsky D. Status of estrogen-androgen balance in hyperthyroid men with Graves' disease. I Clin Endocrinol Metab 1974;38:269-77.

9 Kidd GS, Glass AR, Vigersky RA. The hypothalamicpituitary-testicular-axis in thyrotoxicosis. F Clin Endocrinol Metab 1979;48:798-802.

10 Chopra IJ. Gonadal steroids and gonadotrophins in hyperthyroidism. Med Clin North Am 1975;59:1109-21.

\title{
Lumbar hernia: a rare cause of large bowel obstruction
}

I G Hide, E E Pike, R Uberoi

\section{Summary}

We describe a 70-year-old woman presenting with large bowel obstruction secondary to incarceration of the mid descending colon within a lumbar hernia. This was diagnosed on barium enema and successfully treated surgically.

Keywords: hernia; intestinal obstruction; colon

Lumbar herniation occurs in the region of the flank bounded by the 12 th rib, the iliac crest and the erector spinae and external oblique muscle groups. ${ }^{1}$ Herniation in this area is uncommon with fewer than 300 cases described in the literature. Strangulation is rare. ${ }^{2}$ We describe such a case presenting as distal large bowel obstruction.

\section{Case report}

A 70-year-old woman was admitted with a history of absolute constipation for one week, vomiting and vague lower abdominal pain. A long history of constipation was obtained and the patient reported taking several laxative preparations regularly. There was no history of weight loss. Previous operations included bilateral total hip replacements, appendicectomy and three Caesarean sections. The patient was obese with abdominal distension but no tenderness. No abnormality was found on palpation. Bowel sounds were active but not tinkling. No significant biochemical or haematological abnormalities were detected. An initial diagnosis of constipation with secondary obstruction was made. A plain abdominal film (figure 1) showed features of distal large bowel obstruction with no gas or faecal material seen in the sigmoid colon.

The patient was admitted and treated with suppositories and intravenous fluids and a nasogastric tube was inserted. An instant barium enema revealed sigmoid diverticular disease and a loop of mid descending colon lying lateral to the iliac crest. Both ends of the loop communicated with the remainder of descending colon and the appearances were consistent with a hernia through a narrow orifice (figure 2). A laparotomy was performed during which a moderate-sized hernia through the left inferior lumbar triangle was confirmed. This contained an incarcerated portion of descending colon. The bowel was reduced, found to be viable and the hernial orifice repaired. The patient recovered quickly and was discharged after 7 days.

\section{Discussion}

The lumbar region is anatomically defined superiorly by the lower border of the 12 th rib, inferiorly by the iliac crest, anterolaterally by the posterior border of the external oblique muscle,

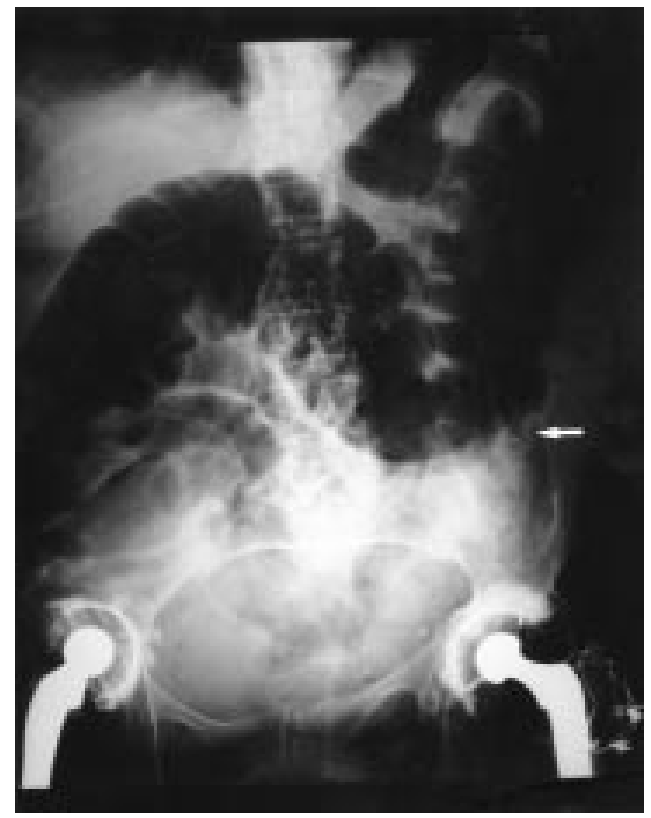

Figure 1 Plain abdominal X-ray demonstrating large bowel obstruction to the mid descending colon (white arrow) 


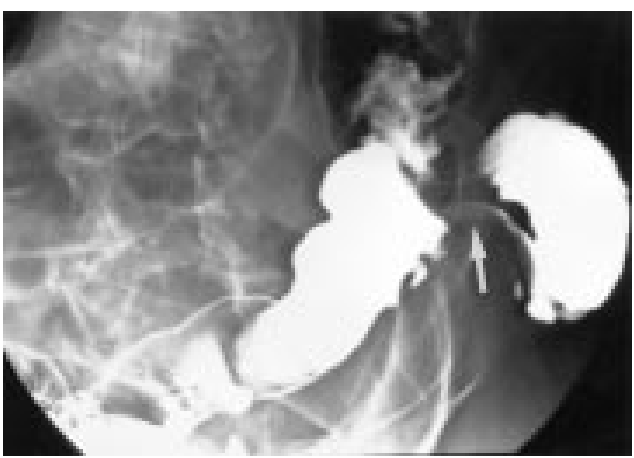

Figure 2 Single contrast barium enema demonstrating a short segment of herniated descending colon lying lateral to the iliac crest. Mucosal folds can be seen passing through the hernial orifice (white arrow)

and posteromedially by the erector spinae muscle group. Hernias can occur anywhere within the region but are most common through the superior lumbar triangle (of GrynfelttLesshaft), an inverted triangle bounded by 12 th rib, erector spinae and the posterior border of the internal oblique muscle. The inferior lumbar triangle (of Petit), bounded by iliac crest, posterior border of external oblique and the anterior border of latissimus dorsi is the next most frequent site. ${ }^{1}$ Herniation in the lumbar region is uncommon with fewer than 300 reported cases. It occurs more commonly in males (ratio 3:1) and is more frequent on the left than the right (ratio 2:1). Patients are usually between 50 and 70 years old. ${ }^{3}$ Lumbar hernias may be acquired $(80 \%)$ or congenital. If acquired, they may be spontaneous $(55 \%)$ or follow trauma, surgery or inflammation $(25 \%)$. Spontaneous herniation is usually the result of raised intra-abdominal pressure and an acquired predisposition such as muscle atrophy due to polio, obesity, old age or debilitating disease. ${ }^{4}$ The hernia may contain retroperitoneal fat, kidney, colon or less commonly small bowel, omentum, stomach, ovary, spleen or appendix. ${ }^{5}$ Patients are usually asymptomatic

1 Geis WP, Saletta JD. Lumbar hernia. In: Nyhus LM, Condon RE, eds, Hernia, 3rd edn. Philadelphia: Lippincott, 1989; pp 401-15.

2 Nyhus LM, Bombeck CT, Klein MS. Hernias. In: Sabiston DC, ed, Textbook of surgery, 14th edn. Philadelphia: Saunders, 1991; pp 1147.

3 Baker ME, Weinerth JL, Andriani RT, et al. Lumbar hernia: diagnosis by CT. AfR 1987;148:565-7.

4 Horovitz IL, Schwarz HA, Dehan A A lumbar hernia presenting as an obstructing lesion of the colon. Dis Colon Rectum 1986;29:742-4.

\section{Learning points}

- lumbar hernias are rare defects in the posterolateral abdominal wall; they may contain fat, kidney, colon or even intraperitoneal structures, most commonly small bowel

- lumbar hernias are a rare cause of bowel obstruction due to incarceration or strangulation of bowel loops within the hernial sac

- lumbar hernias may be difficult to diagnose clinically, and imaging, particularly CT scanning, is helpful

but may complain of low back pain, colic or a pulling sensation. A soft, sometimes reducible mass may be palpable in the flank or bowel sounds audible if the hernia contains intestine. In obese patients detection of a mass is particularly difficult. Bowel incarceration occurs in $25 \%$ but because the hernial neck is generally wide, strangulation is said to be rare. ${ }^{2}$

Recent publications have stressed the role of computed tomography (CT) in the diagnosis of lumbar hernias and elegant demonstration of the anatomy can be obtained. ${ }^{367}$ Unfortunately, many patients have few or no signs to suggest the diagnosis and investigations will usually be tailored to clinical suspicion. In our case, clinical examination and plain abdominal films pointed to distal large bowel obstruction and the findings at barium enema were unexpected, though diagnostic. Lumbar hernias may be detected in this manner if the hernia contains colon but where the diagnosis is suspected, CT remains the investigation of choice as it will demonstrate the hernia regardless of the contents. Recently, reports of successful laparoscopic interventions have been published. ${ }^{8}$

Our case emphasises the clinical diagnostic difficulty posed by lumbar herniation and this condition should be borne in mind as a rare but eminently treatable cause of large bowel obstruction.

5 Thorek M. Lumbar hernia. F Int Coll Surg 1950;14:367-93. 6 Faro SH, Racette CD, Lally JF, et al. Traumatic lumbar hernia. AfR 1990;154:757-9.

7 Lawdahl RB, Moss CN, Van Dyke JA. Inferior lumbar (Petit's) hernia. $A 7 R$ 1986;147:744-5.

8 Heniford BT, Iannitti DA, Gagner M. Laparoscopic inferior and superior lumbar hernia repair. Arch Surg 1997;132: 1141-4. 


\title{
Warfarin-induced skin necrosis
}

\author{
Andrew J Stewart, Ian D Penman, Margaret K Cook, Christopher A Ludlam
}

\begin{abstract}
Summary
Skin necrosis is a rare but serious sideeffect of treatment with warfarin. At particular risk are those with various thrombophilic abnormalities, especially when warfarinisation is undertaken rapidly with large loading doses of warfarin. With the increasing number of patients anticoagulated as out-patients for thromboprophylaxis, we are concerned that the incidence of skin necrosis may increase. If skin necrosis does occur, prompt remedial action may be of benefit in preventing permanent tissue damage.
\end{abstract}

Keywords: warfarin; skin necrosis; protein C; antiphospholipid antibody; adverse drug reaction

Warfarin-induced skin necrosis (WISN) is an uncommon, catastrophic complication of oral anticoagulant therapy. ${ }^{1}$ A substantial minority of cases occur in association with a familial deficiency of protein $\mathrm{C}^{2}$ or protein $\mathrm{S}^{3} \mathrm{An}$ acquired deficiency of protein $\mathrm{S}$ secondary to the development of antiphospholipid antibodies has also been implicated. ${ }^{4}{ }^{5}$ We present three cases of WISN, in two of whom antiphospholipid antibodies were the only identifiable predisposing factor. Neither of these two cases had a low protein $\mathrm{C}$ or $\mathrm{S}$.

\section{Case reports}

Case 1

A 39-year-old woman with no personal or family history of thromboembolic disease suffered a deep vein thrombosis (DVT), confirmed by venography. Treatment was begun with intravenous unfractionated heparin which was continued for 2 days, after which she discharged herself from hospital.

One week later, she was re-admitted with clinical evidence of a pulmonary embolism. Intravenous unfractionated heparin was restarted and warfarin (two doses of $10 \mathrm{mg}$ and one of $5 \mathrm{mg}$ on three consecutive days) was commenced simultaneously. Heparin was discontinued on the third day, when the INR reached 2.3. That evening, she developed extremely painful ecchymotic lesions on both lower limbs. Initially, it was felt that these were haematomas, but over the next 12 hours it became clear that skin necrosis was developing. Despite re-heparinisation and treatment with fresh frozen plasma and intravenous vitamin $\mathrm{K}$, she sustained extensive necrosis of the skin of both legs (figure). She was eventually discharged from hospital 3 months later, after skin grafting. Testing for thrombophilia (table) revealed a lupus anticoagulant and high-titre IgG anticardiolipin antibodies.

\section{Case 2}

A 32-year-old woman had a history of venographically proven puerperal DVT, following which she was anticoagulated with warfarin for about 6 months without complication. Eight years later, she had a second pregnancy. Thromboprophylaxis with subcutaneous unfractionated heparin was begun immediately after delivery. One week later, she developed clinical evidence of a DVT and was warfarinised with simultaneous discontinuation of heparin. Three days after this, a painful 'bruise' appeared on her left thigh, which she had banged on a table. The skin in the centre of the affected area progressed to frank necrosis over the following 48 hours. Warfarin was discontinued, but no other treatment was given. Healing was slow and she required surgical debridement after 7 weeks.

One week after the debridement, she sustained a pulmonary embolism and received intravenous unfractionated heparin. After 48

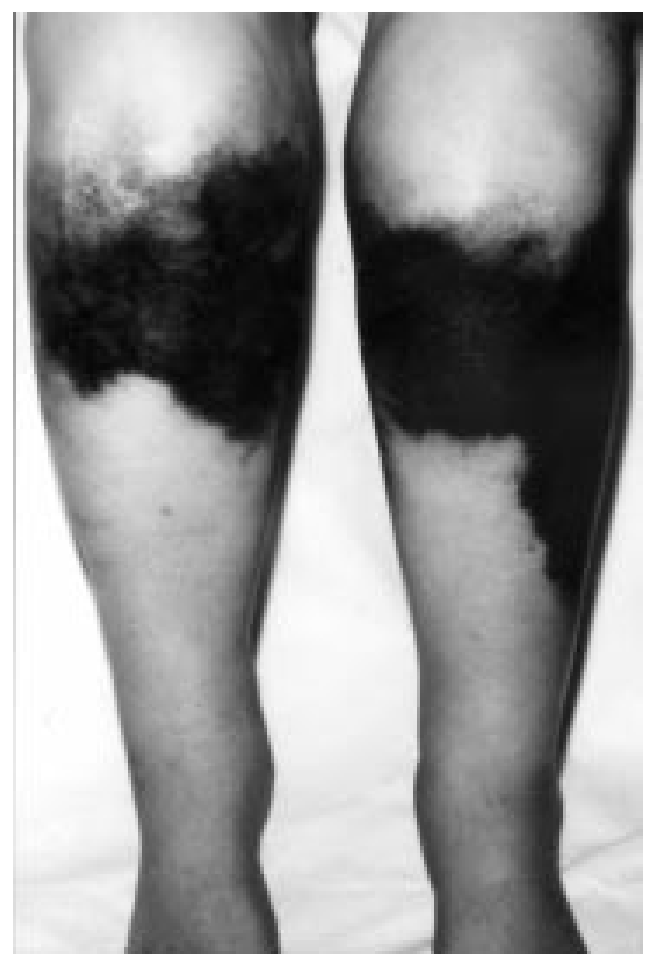

Figure Warfarin-induced skin necrosis affecting both legs 